\title{
WEIGHTED COMPOSITION AND BLOCK SHIFT MATRIX OPERATORS ON $\ell^{2}(\mathbb{N})$ SPACE
}

\author{
M. R. JABBARZADEH AND Z. MOAYYERIZADEH
}

Abstract. In this paper we investigate some clasees of weighted composition operators on the Hilbert space of complex valued functions on the natural numbers. Next we introduce a new model of a block matrix operator $M(\alpha, \beta)$ induced by two sequences $\alpha$ and $\beta$ and characterize its $p$-paranormality. Then we give examples of these operators to show that the $p$-paranormal classes are distinct.

Mathematics subject classification (2010): 47B20, 47B38.

Keywords and phrases: Weighted composition operator, conditional expectation, hyponormal operator, block matrix operator.

\section{REFERENCES}

[1] C. Burnap, I. Jung AND A. LAmbert, Separating partial normality classes with composition operators, J. Operator Theory, 53 (2005), 381-397.

[2] J. CAMPBell And W. Hornor, Seminormal composition operators, J. Operator Theory, 29 (1993), 323-343.

[3] Y. EstaRemi And M. R. JabBaRZADeh, Weighted Lambert type operators on $L^{p}$ spaces, J. Operators and Matrices, 7 (2013), 101-116.

[4] G. EXner, I. JUng AND M. LeE, Block matrix operators and weak hyponormalities, J. Integr. Equ. Oper. Theory, 65 (2009), 345-362.

[5] T. FurutA, Invitation to Linear Operators, Taylor and Francis Inc., 2001.

[6] D. Harrington AND R. Whitley, Seminormal composition operators, J. Operator Theory, 11 (1984), 125-135.

[7] T. Hoover, A. LAmberT And J. Quinn, The Markov process determined by a weighted composition operator, Studia Math. (Poland) LXXII (1982), 225-235.

[8] Y. Cui, H. Hudzik, R. Kumar and L. Maligranda, Composition operators in Orlicz spaces, J. Aust. Math. Soc. 76 (2004), 189-206.

[9] M. R. JabbarZadeh And M. R. AzImi, Some weak hyponormal classes of weighted composition operators, Bull. Korean. Math. Soc. 47 (2010), 793-803.

[10] M. R. JABBARZADEH, A conditional expectation type operator on $L^{p}$ spaces, J. Operators and Matrices, 4 (2010), 445-453.

[11] A. LAMBERT, Hyponormal composition operators, Bull. London Math. Soc. 18 (1986), 395-400.

[12] S. Panayappan, Non-hyponormal weighted composition operators, Indian J. Pure Appl. Math. 27 (1996), 979-983.

[13] M. M. Rao, Conditional measure and applications, Marcel Dekker, New York, 1993.

[14] R. K. Singh And J. S. Manhas, Composition Operators on Function Spaces, North Holland Math. Studies 179, Amsterdam, 1993.

[15] H. TAKAGI, Compact weighted composition operators on $L^{p}$, J. Proc. Amer. Math. Soc. 116 (1992), $505-511$. 